\title{
Determining the Contact Zone in Disk Machining of a Complex Spherical Mill
}

\author{
N. A. Chemborisov, I. Z. Sungatov, and R. M. Khisamutdinov \\ Kamsk State Engineering and Economics Academy, Naberezhnye Chelny
}

DOI: $10.3103 / \mathrm{S} 1068798 X 13040060$

When considering the machining of a helical surface on a sphere, we must determine the contact zone in order to calculate the profile of the generatrix for the initial tool surface. For practical proposes, precise determination of the contact zone - the range of the helical angle $\varphi$-is required. It is sufficient to determine the angular range fully encompassing the contact zone of the helical mill surface and the tool's initial surface of revolution.

The necessary zone of angular projections is equal to the variation of $\varphi$ from angle $\theta_{1}$ to $\theta_{2}$ between the axis $O X$ and the segments connecting point $O$ to the point of intersection of the circumference with an arc in the end cross section of the disk tool (Fig. 1a).

The interaxial distance $A_{\omega i}$, which varies in each cross section, may be determined from the equation

$$
A_{\omega i}=\sqrt{z_{i}^{2}+\left(l+R_{i}\right)^{2}}-h_{i n}+R_{\mathrm{w}} .
$$

In the machining of helical chip channels on a complex spherical mill (Fig. 1b), we must specify the angle $\theta_{\mathrm{mi}}$ of rotation around its own axis, consistent with the other motion $\theta_{\mathrm{mi}}=2 \pi \pm \varphi \tan \omega_{0}+\frac{\tau}{\cos ( \pm \varphi)}$, where $\omega_{0}$ is the inclination of the helical line in the cross section of maximum diameter; $\pm \varphi_{i}$ is the coordinate characterizing the rotation around the vertical axis.

To maintain constant tooth profile and ensure constant mean angle and dimensions of the trough between adjacent teeth, the end of the disk must always be parallel to the resultant linear-velocity vector of the tool's rotation around its own axis and the vertical axis. That is ensured with the following variation in inclination $\omega_{\mathrm{gw}}$ of the grinding wheel

$$
\omega_{\mathrm{gw}}=\arctan \left(\tan \omega_{0} \cos ( \pm \varphi)+\tau \tan ( \pm \varphi)\right) .
$$

Hence, the angle of intersection is $\varepsilon=\pi / 2-\omega_{\mathrm{gw}}$.

The circumference of the end cross section for the initial tool surface of maximum radius may be described by the equation

$$
z^{2}+\left(x-A_{\omega i}\right)^{2}=R_{\mathrm{di}}^{2},
$$

where $R_{\mathrm{di}}=A_{\omega i}-R_{\mathrm{sph}}+h_{i} ; h_{i}$ is the depth of the helical-channel profile.

The equation of the circle obtained in the end cross section is

$$
x^{2}+z^{2}=1 \text {. }
$$

Combined solution of Eqs. (1) and (2) yields the coordinates of their intersection

$$
\left.\begin{array}{l}
z^{2}+\left(x-A_{\omega i}\right)^{2}-R_{\mathrm{di}}^{2}=0 ; \\
x^{2}+z^{2}-1=0 .
\end{array}\right\}
$$

Transformation of Eq. (3) yields $1-2 x A_{\omega i}+A_{\omega i}^{2}-$ $R_{\mathrm{di}}^{2}=0$. The solution of that equation is

$$
\left.\begin{array}{l}
x_{1,2}=A_{\omega i} \pm R_{\mathrm{di}}^{2} ; \\
z_{1,2}= \pm \sqrt{1-x_{1,2}^{2}} .
\end{array}\right\}
$$

Hence, we may determine $\theta_{1}$ and $\theta_{2}$ from the formulas $\theta_{1}=\arctan \left(z_{1} / x_{1}\right) ; \theta_{2}=\arctan \left(z_{2} / x_{2}\right)$.

As a result, we obtain the interval corresponding to the contact zone of the disk and tool surfaces. On that basis, the scope of the subsequent calculations may be determined.

(a)
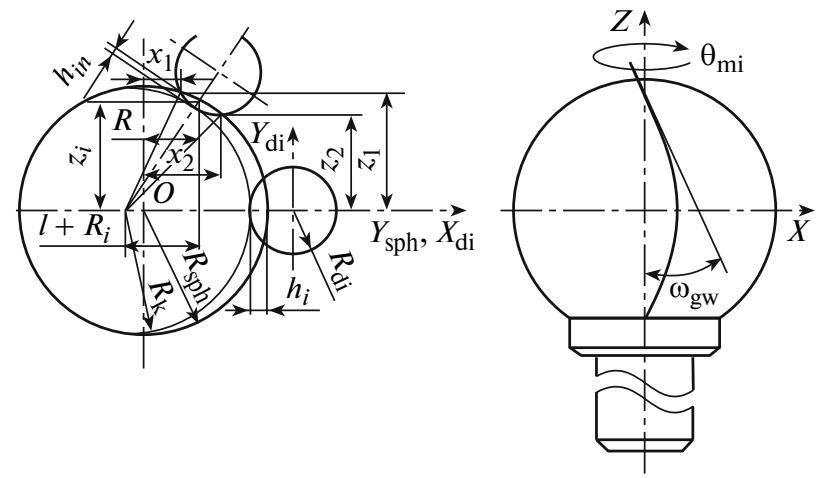

Fig. 1. Determining the range within which the position of the contact zone is to be determined (a); machining of helical chip channels by means of a complex spherical mill (b). 